\title{
Nano-black carbon (biochar) released from pyrogenic carbonaceous matter as a super suspending agent in water/soil environments
}

\author{
Fei Lian $^{1} \cdot$ Zhenyu Wang $^{1} \cdot$ Baoshan Xing ${ }^{2}$
}

Received: 2 September 2020 / Accepted: 30 October 2020 / Published online: 23 November 2020

(c) The Author(s) 2021, corrected publication 2021

\begin{abstract}
Nano-black carbon (BC) is one of the most active fractions in the pyrogenic carbonaceous matter continuum. The majority of recent studies mainly focus on the role of nano-BC in the global carbon cycle. However, based on literature and our recent studies, we suggest that nano-BC may also serve as a super suspending agent, carrier, and redox mediator for sorbates during its migration from terrestrial to water bodies due to its unique properties such as high colloidal stability, strong sorption capacity, and high surface reactivity. The full implications of nano-BC in water/soil environments are far more than we expected. Thus, we call for more detailed investigations on the activity and reactivity of nano-BC in water/soil environments.
\end{abstract}

Keywords Biochar $\cdot$ Nanoparticle $\cdot$ Suspension $\cdot$ Sorption $\cdot$ Redox capacity $\cdot$ Biogeochemical processes

Pyrogenic carbonaceous matter (PCM) such as charcoal, soot, and biochar is generally produced by incomplete combustion of fresh and/or fossilized biomass, which is a significant fraction of natural organic matter (NOM) and ubiquitous in water/soil environments. Besides vegetation fires (e.g., forest fires and crop residue burnings), man-made PCM products (mainly biochar) are being produced much more than ever before for various environmental and agricultural applications. Statistically, the global production of biochar can be up to $270 \mathrm{Tg}$ per year, and majority of the products would remain in soils (Jha et al. 2010). The environmental functions of biochar are based on the assumption that it represents one of the most inert natural organic $\mathrm{C}$ pools and can be stored in soils/sediments at centurial or millennial time scales. However, an increasing number of studies have demonstrated that a fraction of PCM can be mobilized into aquatic ecosystems in dissolved and colloidal forms, serving as a crucial contributor to the global C flux.

\footnotetext{
Zhenyu Wang

wang0628@jiangnan.edu.cn

$\triangle$ Baoshan Xing

bx@umass.edu

1 Institute of Environmental Processes and Pollution Control, and School of Environment and Civil Engineering, Jiangnan University, Wuxi 214122, China

2 Stockbridge School of Agriculture, University of Massachusetts, Amherst, MA 01003, USA
}

More importantly, the nano-sized black carbon (nano-BC) has extraordinary suspending ability in aquatic environments, which can strongly sorb contaminants and nutrients (e.g., $\mathrm{N}$ and $\mathrm{P}$ ), heteroaggregate with minerals, and mediate environmental transformation of certain compounds. Consequently, the full implications of nano-BC in water/ soil environments are far more than we expected. Here, we suggest that nano-BC is not only a soil $\mathrm{C}$ pool but a super suspending agent, greatly altering the transport and behavior of elements, chemicals, and soil components (e.g., minerals, NOM, and organisms) in water/soil environments.

Nano-BC has an outstanding colloidal stability in aqueous solutions, which is reflected by its much higher critical coagulation concentration (Fig. 1a) relative to that of engineered carbon nanomaterials [such as carbon nanotube (Saleh et al. 2008), fullerene, graphene oxides (Sun et al. 2018)] and soil colloids (e.g., iron oxides). Moreover, the unique properties of nano-BC including abundant oxyl groups, large surface area, and high reactivity (Lian et al. 2019) endow them with higher sorption affinity for contaminants (especially hydrophobic organic compounds) than NOM (Fig. 1b) (Yue et al. 2019). Thus, nano-BC is expected to greatly enhance the suspension and transport of contaminants, as well as alter the distribution of contaminants among solid phases and solutions, influencing their environmental risks. Nano-BC also has high sorption affinity for biomacromolecules including proteins, extracellular DNA, and even bacterial cells through multiple interactions (e.g., electrostatic interactions, $\pi-\pi$ 

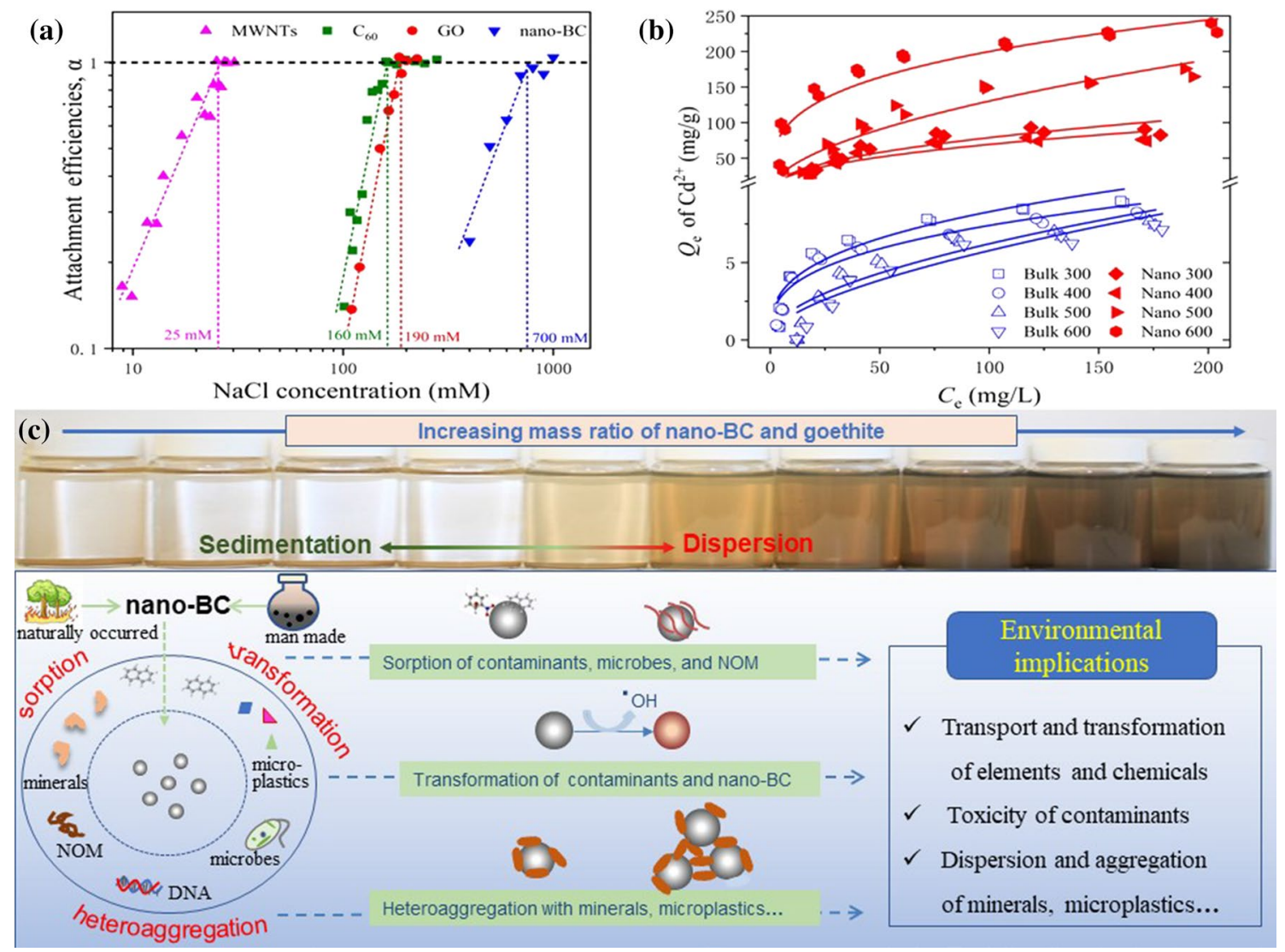

Fig. 1 Schematic diagram for the effects of nano-BC as a suspending agent on the transport and behavior of contaminants and solid fractions in water/soil environments because of its higher dispersion

stacking, hydrogen bonding, and covalent conjugation) (Lian et al. 2020), which is rarely reported in the literature. Potentially, nano-BC can be considered as an important carrier of contaminants and a linker between chemical and biological processes in water/soil systems (Qian et al. 2016). Thus, the relevant mechanisms of nano-BC in sorption/desorption, co-migration, and reactions with contaminants, biotic and abiotic components in soils merit further studies for better understanding the role of nano-BC in these processes.

Nano-BC readily heteroaggregates with oppositely charged soil colloids by electrostatic attraction. For example, nano-BC could significantly enhance the dispersion of goethite via heteroaggregation when its concentration was higher than the critical concentration (Fig. 1c) (Lian et al. 2019). The dispersed goethite may possess unprecedented properties because of the binding of nano-BC, such as increased sorption affinity to contaminants and charge reversal. For like-charge and neutral colloids, nano-BC could also combine with them through other mechanisms such as core-shell stabilization (Peng et al. 2015). The increased loading of nano-BC (natural or man-made) into soils indicates that heteroaggregation of nano-BC with natural ability (a), sorption for contaminants (b), and attachment tendency (c). Data were from Saleh et al. (2008), Sun et al. (2018), Lian et al. (2019), and Yue et al. (2019)

colloids is a widespread phenomenon, however, the related impacts on the composition, structure, and reactivity of indigenous soil colloids are largely unknown. Heteroaggregation between nano-BC and soil colloids can be considered as an ordered assembling process, mainly controlled by the solution chemistry ( $\mathrm{Li}$ et al. 2017). The information on the configuration, stability, activity, and reactivity of the ordered assemblies is crucial to fully evaluate their environmental behaviors. From an application perspective, nano-BC is also important for the design of in-situ remediation materials for contaminated soils and groundwater.

The abundant oxyl groups and graphitic structures imply that nano-BC is not only a passive sorbent but a redox-active material. However, relative to bulk-PCM, the catalytic ability of nano-BC is much less identified and understood. We recently observed that nano-BC can trigger decomposition and transformation inhibition of extracellular DNA owing to oxidative damage (Lian et al. 2020), which is among the first to shed light on the reactivity of nano-BC. In addition, other attached chemicals, biomacromolecules, and colloidal particles are likely to experience transformation via electron transfer with nano-BC. Further, nano-BC is photoactive and 
can generate reactive oxygen species during sunlight irradiation (Fu et al. 2016), which could greatly alter the properties and reactivity of nano-BC. Hence, we suggest that besides sorption and aggregation, nano-BC-induced transformation should be paid more attention, which is probably one of the most important driving forces to regulate the fate and transport of elements, chemicals, engineered nanoparticles, and microorganisms in the environment. Due to the extremely small particle size and high surface reactivity, nano-BC might cause potential toxicity to microbiota and plants; however, the relevant knowledge is very limited. Furthermore, the presence of NOM would unavoidably affect the properties, heteroaggregation, and reactivity of nano-BC, which also deserves detailed investigations.

Nano-BC is one of the most active fractions in the PCM continuum. Previous studies mainly focus on the role of nano-BC in the global carbon cycle. It is also worth noting that nano-BC may serve as a suspending agent, carrier, and/ or mediator for sorbates during its migration from terrestrial to water bodies, which has profound implications for many critical biogeochemical processes (Fig. 1). For example, if the effect of nano-BC on the transport of contaminants is not considered in theoretical predicting models, it would probably lead to systematic errors in evaluating their distribution and fate in the soil/aquatic systems. Thus, more effort should be made to the sorption, heteroaggregation, and reactions of nano-BC with anthropogenic contaminants (e.g., organic compounds, metals, engineered nanoparticles, microplastics) and solid fractions (e.g., natural colloids, NOM, organisms). On the other hand, due to the high suspending ability and in situ reactivity, nano-BC would be a promising candidate for designing smart remediation materials, nano-fertilizers, and nano-pesticides in the future environmental and agricultural applications.

Acknowledgements The work was supported by the National Natural Science Foundation of China (41977278 and 41573127) and the Fundamental Research Funds for the Central Universities (JUSRP22015). BX acknowledges the UMass Amherst Conti Faculty Fellowship.
Open Access This article is distributed under the terms of the Creative Commons Attribution 4.0 International License (http://creativeco mmons.org/licenses/by/4.0/), which permits unrestricted use, distribution, and reproduction in any medium, provided you give appropriate credit to the original author(s) and the source, provide a link to the Creative Commons license, and indicate if changes were made.

\section{References}

Fu H, Liu H, Mao J, Chu W, Li Q, Alvarez PJJ, Qu X, Zhu D (2016) Photochemistry of dissolved black carbon released from biochar: reactive oxygen species generation and phototransformation. Environ Sci Technol 50:1218-1226

Jha P, Biswas AK, Lakaria BL, Rao AS (2010) Biochar in agricultureprospects and related implications. Curr Sci 99:1218-1225

Li QQ, Chen BL, Xing BS (2017) Aggregation kinetics and self-assembly mechanisms of graphene quantum dots in aqueous solutions: cooperative effects of $\mathrm{pH}$ and electrolytes. Environ Sci Technol 51(3):1364-1376

Lian F, Yu WC, Wang ZY, Xing BS (2019) New insights into black carbon nanoparticle-induced dispersibility of goethite colloids and configuration-dependent sorption for phenanthrene. Environ Sci Technol 53:661-670

Lian F, Yu WC, Zhou QX, Gu SG, Wang ZY, Xing BS (2020) Size matters: nano-biochar triggers decomposition and transformation inhibition of antibiotic resistance genes in aqueous environments. Environ Sci Technol 54:8821-8829

Peng Y, Pignatello JJ, Uchimiya M, White JC (2015) Heteroaggregation of cerium oxide nanoparticles and nanoparticles of pyrolyzed biomass. Environ Sci Technol 49:13294-13303

Qian LB, Zhang WY, Yan JC, Han L, Gao WG, Liu RQ, Chen MF (2016) Effective removal of heavy metal by biochar colloids under different pyrolysis temperatures. Bioresour Technol 206:217-224

Saleh NB, Pfefferle LD, Elimelech M (2008) Aggregation kinetics of multiwalled carbon nanotubes in aquatic systems: measurements and environmental implications. Environ Sci Technol 42:7963-7969

Sun B, Zhang Y, Chen W, Wang K, Zhu L (2018) Concentration dependent effects of bovine serum albumin on graphene oxide colloidal stability in aquatic environment. Environ Sci Technol 52:7212-7219

Yue L, Lian F, Han Y, Bao QL, Wang ZY, Xing BS (2019) The effect of biochar nanoparticles on rice plant growth and the uptake of heavy metals: Implications for agronomic benefits and potential risk. Sci Total Environ 656:9-18 